\title{
Mechanical circulatory support in congenital heart surgery: Are we there yet?
}

\author{
Mark Rodefeld, MD
}

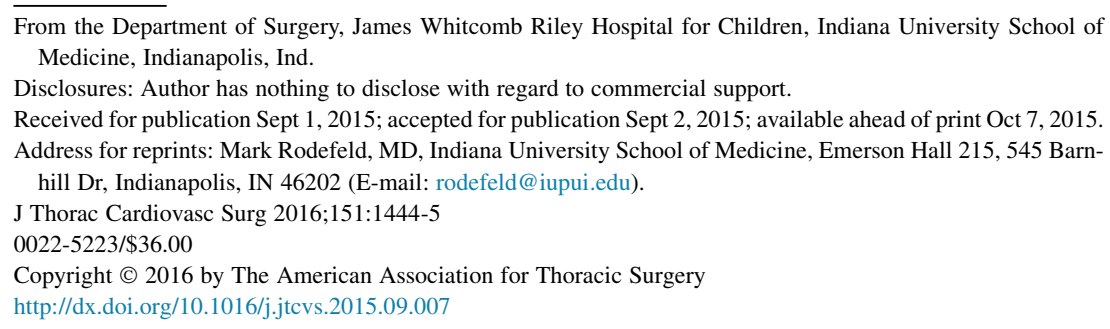

Several mechanical circulatory support (MCS) devices are currently available for use in children, but the choices are limited and based on older technology. These devices are associated with high morbidity rates, which limits or makes their use unfavorable. The impetus for development of MCS devices has been adult heart failure. The use of these devices in pediatric applications may result in patientdevice (size or functional) mismatch. Pervasive to nearly all pediatric device applications, this clouds the perceived likelihood of success for devices applied in children. CurCurrent third-generation MCS technology is evolving toward smaller continuous-flow bearingless rotary pumps. This promising trend is likely to increase device applicability in adolescents and children; it is less clear to what extent neonate and infant applications may be affected. Historically, most MCS applications in children have originated from adult devices. Conversely, it is not the case that MCS devices have been developed expressly for congenital heart disease. It is in the latter approach where significant innovations are likely to arise.

There currently are no fully implantable blood pumps for children, whereas there are several for adults. The promise of biologic therapies for cardiac replacement (eg, mitigation of chronic rejection, xenograft and stem cell therapies, growing new hearts) has yet to be realized. Until such a time, a significant need for chronic MCS devices specific for congenital heart disease will persist. As technology improves and morbidity decreases, the indications and uptake for MCS in children will inevitably expand. This is good news, because many pediatric patients have high potential for functional recovery of their cardiac or pulmonary insufficiency and stand to benefit.

\section{THE POTENTIAL FOR FUTURE RESEARCH}

There is a significant need for MCS devices tailored specifically to congenital heart disease. However, the relatively small pediatric market size and perceived complexity of disease make this extremely challenging. Smaller devices that are fully implantable, have low power consumption,

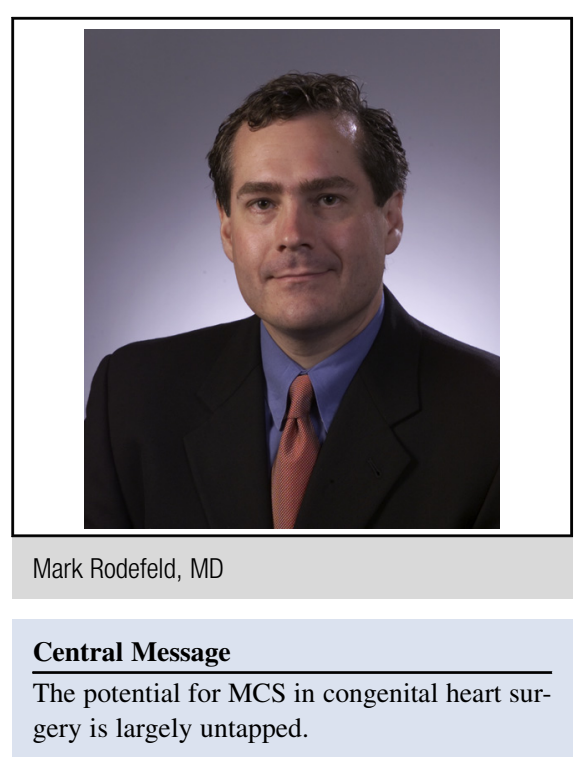

See Article page 1730

See Editorial Commentary page 1737.

and can be powered transcutaneously without externalized drivelines obviously would be of significant value. Fortunately, MCS technologies are moving in this direction.

To move the field forward, it will be necessary to innovate new devices that are specifically designed to address unique pediatric circulatory needs. This is a daunting challenge. New devices may vary significantly from existing technologies, must scale to smaller sizes, must be indifferent to growth potential, and must have long-term durability. Underlying these issues, as with all extracorporeal circulatory therapies, is the need to mitigate the inflammatory response to nonbiologic surfaces and improve thrombogenicity profiles. With new technologies may come unexpected new problems. An example is acquired von Willebrand's disease, which seems to be related to blood component exposure to shear stresses in the thin-film blood gap of bearingless devices.

For certain congenital heart diseases, entirely new device designs applied in new locations or new physiologic roles may need to be considered. This is interesting and of high potential value because the targeted development of a device itself may enable entirely new therapeutic approaches. With this in mind, patients with a single functional ventricle 
may stand to benefit the most. For the increasing number of patients palliated with Fontan circulations, their therapeutic options are limited and their inherent circulatory inefficiency will ultimately culminate in failure. Concepts are evolving that make right-sided circulatory support of the univentricular Fontan circulation a realistic consideration. A device that can safely and reliably provide cavopulmonary assist in a Fontan circulation can in theory emulate a biventricular circulation, preventing insidious and persistent collateral disease (heart, liver, intestines) and significantly improving quality of life. Although cavopulmonary assist may be the key to reverse or prevent Fontan circulatory inefficiency, such a device does not yet exist. Until one becomes available, reversal of the Fontan circulation will remain an impossibility (short of transplantation).

\section{CHALLENGES FOR THE FUTURE}

Congenital heart surgeons need devices that are specific for use in children and adults with congenital heart disease, including both systemic and pulmonary support devices. The lack of traditional commercialization incentives to develop these technologies make significant innovation a difficult and nearly improbable task. So, how can the field move forward?

Our focus perhaps should be on developing devices specific to patient needs, rather than on modification of existing devices. As early-stage ideas evolve to the point of appearing safe and reliable, funding sources are likely to be found to support further development. The trick for the innovator is how to reach this point. Referred to as the "valley of death," it is difficult to cross the chasm from idea to a commercializable product; for pediatric applications, the difficulty is an order of magnitude greater than for larger market share devices. It may require the philanthropic support of concerned stakeholders. Congenital heart disease foundations may have the potential to engage in mission investing, with surprising impact. A recent example is the Cystic Fibrosis Foundation, which successfully supported the development of a promising new drug therapy while simultaneously supporting its mission.

Support for pediatric MCS device development is available through various federal funding mechanisms. Significant funding has just been completed in the prior decade for the development of pediatric blood pumps; the difficulty in realizing the objective of this initiative highlights the complexity involved. The National Institutes of Health recently issued an important and highly relevant initiative under the Topics of Special Interest for the Small Business Innovation Research Program. ${ }^{1}$ It calls for the "Development of mechanical circulatory support devices for individuals with congenital heart disease and single ventricle physiology after Fontan surgical palliation." Other incentives exist via orphan device regulatory pathways to aid commercialization of pediatric MCS devices. Although these initiatives are extremely important, perhaps even greater incentives should be considered because the barriers are so high.

Future applications of MCS device therapy in congenital heart disease will redefine destination therapy. In adults, destination therapy may imply end-stage therapy where no other options are available. For children who may otherwise have decades of life expectancy ahead of them, and tissues that are otherwise physiologically healthy beyond their structural deficits, destination therapy may mean decades of survival beyond the service of the device. Fully implantable systems must be designed so that they have minimal or no detriment if they ever cease to function. Until some of the current limitations with device technology are overcome (thrombogenicity, energy requirement, transcutaneous energy transfer), the development of new devices specific to congenital heart disease may remain an elusive goal. However, we should not be deterred. By increasing awareness, encouraging acceptance of new technologic approaches, and advocating for reduction of commercialization barriers, the field will be successful in developing badly needed new technologies.

So, are we there yet? We aren't even close. We are at an early stage using devices that are likely, in retrospect, to appear primitive and shortsighted. Refinement of existing technologies will produce incremental improvements, but the biggest advances will likely come from innovation breakthroughs that vary significantly from existing approaches. It will take courage for surgeon-scientists to pursue blue sky, out-of-the-box approaches. Such endeavors should be encouraged and supported. I am optimistic and see a bright future for specialized device therapy for congenital heart disease in the coming decades. It is here where the field may have its greatest challenges and most exciting potential for advances.

\section{Reference}

1. National Heart, Lung, and Blood Institute. Small business topics of special interest. Notice number NOT-HL-15-266. July 10, 2015. Available at: http://grants.nih. gov/grants/guide/notice-files/NOT-HL-15-266.html. 